\title{
Editorial
}

\section{Not Just Another Guideline}

\author{
James T. Lee, MD, PhD
}

This issue of Infection Control and Hospital Epidemiology provides a full reproduction of the recently completed "Guideline for the Prevention of Surgical Site Infection, 1999," a sizable document issued by the Centers for Disease Control and Prevention (CDC) through the combined efforts of its staff professionals and its 12 member Hospital Infection Control Practices Advisory Committee (HICPAC)..$^{1}$ I was privileged to be the sponsoring member for this new guideline, with the opportunity to designate outside experts from the disciplines of surgery, surgical nursing, epidemiology, and hospital infection control. The outside experts were given early access to the guideline in its primitive draft stage, and their comments and advice were taken into consideration during formal HICPAC deliberations. In accordance with $\mathrm{CDC}$ policy and federal regulations, a working draft of the guideline was published in the Federal Register for the purpose of eliciting public commentary during a 60 -day period. While the finetuning of the guideline involved very focused revision efforts of a few persons, including me, it should be made clear that $\mathrm{CDC}$ staff did the hard scut work: background literature searching and first draft production; numerous chores of draft printing, collating, and distribution; and a final, line-by-line inspection of the finished product.

Surgical-site infection (SSI) is one of the best-known surgical outcome flaws, and every operation, regardless of specialty, carries a finite risk of incisional SSI (formerly called "wound infections"), as well as deep (organ/space) SSI (eg, postoperative peritonitis, mediastinitis, etc). In modern care, the likelihood of SSI is usually very low, but nobody disputes that the prevention of SSI has economic, clinical, patient satisfaction, and patient functional status value. Additionally, it can be argued that the approach to reduction of SSI risk in a given practice is a generally instructive model for surgical-care quality improvement of any type. The sequence of steps used for care quality improvement involving SSI risk reduction has been described in detail..$^{2}$ There must be ongoing, accurate accrual of observational data; skillful interpretation of both numerical and factual information, with attention to SSI risk heterogeneity and the meaning of secular variation patterns; and cautious but incremental "process changes" when (and only when) patterns of past SSI data are persuasive. Of fundamental importance is the availability of basic knowledge concerning "what works" to achieve SSI risk reduction in various clinical contexts. Also, it recently has become more obvious to some surgeons and epidemiologists that efforts to understand, design, and apply SSI prevention strategies tend to be confusing, ineffectual, or even self-defeating unless there is attention to basic tenets of analytical epidemiology, keen awareness that SSI involves multiple pathophysiological variables, and acceptance of the fact that no known SSI prevention measure is completely effective.

To address SSI prevention comprehensively, the CDC has produced the guideline found in this issue. In the first part of the guideline, an overview of SSI phenomenology is provided. There the user will find extensively referenced discussions of SSI terminology, microbiology, pathogenesis, adjunctive preventive measures, epidemiology, and surveillance. Various details of patient care that directly alter SSI risk are surveyed in the text according to the chronological order in which they occur during actual surgical-patient care. For example, the discussions of hand scrubbing, gloving, and surgical gowns precede comments on surgical technique; details of intraoperative asepsis are covered before addressing the postoperative care of fresh incisions.

In its second part, the guideline supplies numerous carefully articulated recommendations for SSI risk reduction. Each recommendation carries a category label; final consensus regarding such labeling was achieved during formal HICPAC meetings. As one who was "in the room," I can certify that there were frank arguments, extensive discussion, and intensive dialectic. In sum, category I recommendations are 
effective measures advanced by HICPAC as the best available (but not necessarily perfect) advice. The CDC position is clear: all category I recommendations should be adopted by all healthcare facilities. The subgroup labels IA and IB were used to differentiate quality of evidence, with IA nominally indicating that reliable scientific evidence was located and reviewed by HICPAC. The label IB was used to specify that available evidence was weaker than that used for making IA recommendations, usually because controlled clinical studies were not discoverable. Category II status was given to recommendations for SSI risk reduction tactics that are not fully supportable by "good science" but that nevertheless have appeal based on theoretical principles or multiple observational accounts provided by seasoned surgical experts. Finally, a few unproved practices that have become part of the lore of SSI prevention were deemed "unresolved."

The new guideline replaces two rather brief prior CDC documents ${ }^{3,4}$ that dealt with SSI, and it has obvious strengths not found in those predecessors. For example, the user will find an enormous bibliography (497 citations), a list of selected readings (21 books, book chapters, or articles judged as seminal), and 10 richly detailed tables. There is also a learner-paced study package at the end of the guideline; anyone may take the multiple-choice examination and submit answers to the $\mathrm{CDC}$ to receive continuing education credit. An important contribution of the guideline is its indirect explication of the consequences of misconstruing the epidemiological term risk factor, perhaps one of the most frequently (and inexcusably) misused terms in many textbooks and papers that deal with surgical outcome flaws of all types (my fellow surgeons are often the culprits, but not always). There is also a first-rate discussion of many practical details of SSI surveillance methodology, with clear explanations of what to do and how to do it. Surveillance data-gathering pitfalls are mentioned, some subtle and others "no-brainers." These are reflective of some bittersweet lessons learned during recent years in large centers that perform global infection surveillance, ${ }^{5}$ as well as in the National Nosocomial Infection Surveillance effort at the CDC.

An old cliché teaches that a camel is a horse designed by a committee, but we should not be too quick to assign "camel status" to the new SSI guideline. On behalf of HICPAC, it bears repeating that numerous experts from various fields besides surgery had input and provided both supportive and alternative views to the $C D C$. Notwithstanding HICPAC efforts to find and shape consensus regarding issues in the complex-and often murky-backwaters of recent SSI scholarship, it is very unlikely that there will be no criticisms of either the foundational text of the guideline or some of its recommendations. If post hoc criticism stimulates productive thinking, forces the reconsideration of classical articles, or triggers the construction of potentially disprovable hypotheses that lead to new clinical trials of SSI prevention modalities, the guideline will have had an unanticipated, but entirely satisfying, impact. It is my view that the new SSI guideline, rather like a dependable horse, will be a valuable companion on the journey to excellence in one area of surgical-outcome improvement.

\section{REFERENCES}

1. Mangram AJ, Horan TC, Pearson ML, Silver LC, Jarvis WR, the Hospital Infection Control Practices Advisory Committee. Guideline for prevention of surgical site infection, 1999. Infect Control Hosp Epidemiol 1999;20:247-280.

2. Lee J. Surgical wound infections: surveillance for quality improvement. In: Fry DE, ed. Surgical Infections. Boston, MA: Little Brown \& Co; 1995:145-159.

3. Simmons BP. Guideline for the prevention of surgical wound infections. Infect Control 1982;3:188-196.

4. Garner JS. Guideline for the prevention of surgical wound infections. Infect Control 1986;7:193-200.

5. Lee JT, Olson MM. Wound infection surveillance for 85,260 consecutive operations. Journal of Surgical Outcomes. In press. 


\section{PROTECT YOURSELF FROM NEEDLESTICKS}

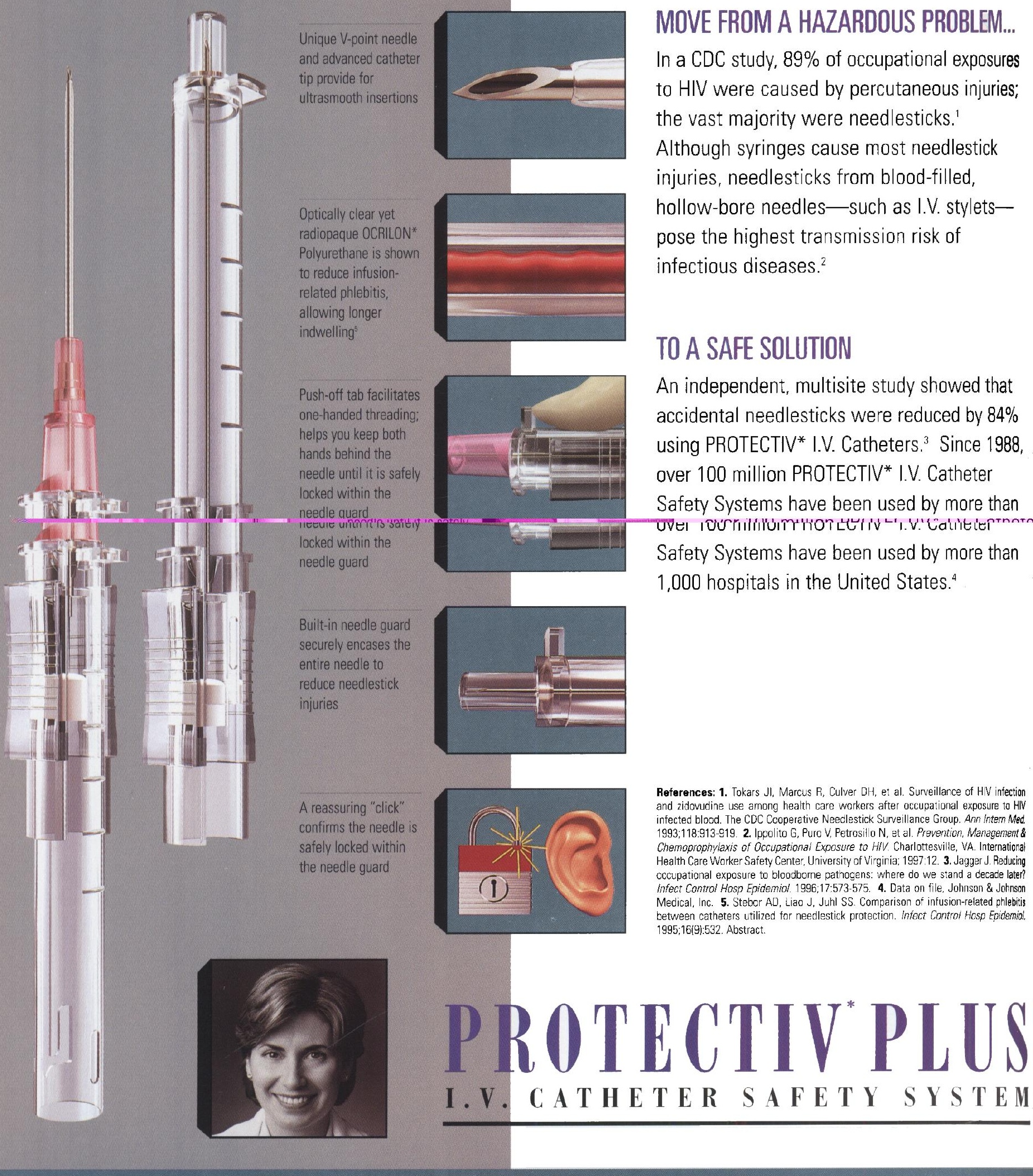

